\title{
NATURAL AXIOMS FOR CLASSICAL MEREOLOGY
}

\author{
A. J. Cotnoir and Achille C. Varzi
}

\begin{abstract}
We present a new axiomatization of classical mereology in which the three components of the theory-ordering, composition, and decomposition principles - are neatly separated. The equivalence of our axiom system with other, more familiar systems is established by purely deductive methods, along with additional results on the relative strengths of the composition and decomposition axioms of each system.
\end{abstract}

As a formal theory of parts and wholes, mereology should tell us three sorts of thing. It should say:

(i) what sort of relation parthood is;

(ii) what sorts of condition govern mereological composition, i.e., intuitively, what it takes to form a whole by "adding things" together;

(iii) what sorts of condition govern mereological decomposition, i.e., intuitively, what happens when we "subtract things" from a given whole.

Classical mereology-the theory stemming from the work of Leśniewski [3] and of Leonard and Goodman [2] - provides a clear answer to each of these questions. It answers (i) by taking parthood to be a partial order (i.e., a reflexive, transitive, antisymmetric relation); it answers (ii) by taking composition to be unrestricted (so that adding any number of things together, no matter how disparate and gerrymandered those things might be, always yields a further thing); and its answers (iii) by taking decomposition to be fully subtractive (so that the mereological difference between any two things, when it exists, always leaves an exact remainder). Interestingly, however, none of the extant axiomatizations of classical mereology does the job explicitly. All are explicit about (ii), and all are (more or less) explicit about (i), modulo redundancies. When it comes to (iii), however, the answer usually comes as a theorem. That is, the axioms do not quite address the question directly but 
rather tell us something else, typically in the form of a supplementation principle, from which the answer follows. There is, of course, nothing wrong with this way of proceeding. Yet it would be more natural and theoretically more elegant if in each case the answer could be captured directly (and non-redundantly) by means of suitable axiom(s). In this note we offer a new axiomatization of classical mereology that does just that.

In section 1, we introduce our axiom system and explain how the axioms give clear and natural answers to questions (i), (ii), and (iii). In section 2, we explore more deeply the connection between composition and decomposition, providing a formal argument to the effect that they are 'two-sides of the same coin'. This serves to illustrate the connections between (ii) and (iii) displayed by our axiomatization. In Section 3 we prove the equivalence of our system with other, more familiar axiom systems by purely deductive methods. We conclude in Section 4 with some additional remarks on the relative strengths of the composition and decomposition axioms of each theory vis à vis our initial questions.

\section{THE AXIOM SYSTEM CM}

We assume a standard first-order language with identity supplied with a distinguished binary predicate constant, $P$, to be interpreted as the parthood relation. The underlying logic is the classical predicate calculus.

To introduce our axiom system for classical mereology, CM, we begin with some definitions.

(D.1) $P P x y: \equiv P x y \wedge x \neq y$

Proper Parthood

(D.2) $O x y: \equiv \exists z(P z x \wedge P z y)$

Overlap

(D.3) $F_{\varphi} x: \equiv \forall z(\varphi \rightarrow P z x) \wedge \forall y(\forall z(\varphi \rightarrow P z y) \rightarrow P x y)$

Fusion

D.1 is a standard definition to the effect that a proper part is any part distinct from the whole. D.2 simply states that things overlap whenever they have at least one part in common. As for D.3, from the standpoint of mereology this is a slightly unusual definition of the fusion predicate, which is meant to capture the notion of something being composed of a specified collection of things.[? It is, however, straightforward and intuitive from the perspective of lattice theory and algebra. Where $\varphi$ is any open formula with just $z$ free, $F_{\varphi} x$ says that $x$ is a minimal upper bound, relative to $P$, of the objects satisfying $\varphi$ : the first conjunct states that $x$ is an upper bound of the $\varphi$ s, while the second states any (other) upper bound includes $x$ as a part.

\footnotetext{
${ }^{1}$ The usual alternate definition, $P P x y: \equiv P x y \wedge \neg P y x$, is equivalent in this system.

${ }^{2}$ For other definitions, see e.g. [1] and [?, \$4].
} 
Now for the axioms.

(A.1) $\forall x P x x$

\author{
Reflexivity \\ Antisymmetry \\ Transitivity \\ Unrestricted Fusion \\ Remainder
}

(A.2) $\forall x \forall y((P x y \wedge P y x) \rightarrow x=y)$

(A.3) $\forall x \forall y \forall z((P x y \wedge P y z) \rightarrow P x z)$

(A.4) $\exists z \varphi \rightarrow \exists x F_{\varphi} x$

(A.5) $\forall x \forall y(\neg P x y \rightarrow \exists z \forall w(P w z \leftrightarrow(P w x \wedge \neg O w y)))$

The first three axioms, A.1, A.2, and A.3, state that $P$ is a weak partial order. Together with D.1, they entail that $P \boldsymbol{P}$ is the corresponding strict partial order. These axioms constitute CM's answer-the standard answer-to (i).

The next axiom, A.4, is actually an axiom schema. It states, for any satisfiable open formula $\varphi$ with just $z$ free, that a fusion of the $\varphi$ s exists. This axiom schema constitutes CM's answer to (ii) and, again, it is in the spirit of the standard answer (modulo any discrepancies concerning the exact definition of 'fusion'): any specifiable non-empty collection of things compose something. It is easily seen from A.2 that $F_{\varphi} x$ is always unique. So, the upper bounds that exist according to A.4 are least upper bounds, corresponding to the familiar lattice-theoretic notion of supremum.

The last axiom is, when it is discussed at all, referred to as the Remainder Principle, and is usually derived as a theorem. [1 states that whenever $x$ is not itself part of $y$, there is always something-a remainder of $y$ in $x$-that has as parts all and only those parts of $x$ that don't overlap $y$. Again, it follows immediately from A.2 (and A.1) that such a remainder must be unique, so A.5 is truly a decomposition principle in the sense of (iii); it tells us exactly what is left when $y$ is "subtracted" from $x$. Of course, if $x$ were part of $y$ there wouldn't be anything left of $x$ were $y$ to be subtracted, and this explains the antecedent of A.5. When the antecedent applies, however, we know from A.1 that $x \neq y$, and there are three different cases. It may be that $y$ is a proper part of $x$, in which case the remainder would just be $x$ without $y$. Or $x$ and $y$ may properly overlap, and hence the remainder would be the biggest part of $x$ that doesn't overlap $y$, namely $x$ minus all the parts it has in common with $y$. Or $x$ and $y$ may have no parts in common at all, in which case subtracting $y$ from $x$ would leave $x$ just as it is. Each of these scenarios is represented in Figure 1, where the shaded area represents the remainder of $y$ in $x$.

\footnotetext{
${ }^{3}$ See e.g. [4, p. 89].

${ }^{4}$ Some readers will be reminded of the notion of set difference, $X \backslash Y$, namely the set of all members of $X$ that are not members of $Y$. In mathematics, these sorts of remainders are also called relative complements, and the same notion is familiar from logic: where $p$ and $q$ are propositions, the relative complement of $q$ in $p$ is the proposition $p \wedge \neg q$.
} 

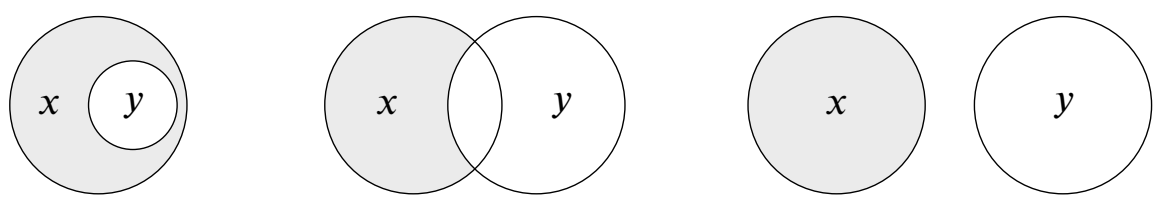

Figure 1: Cases where $\neg P x y$

\section{RESIDUATION}

We have seen informally that A.5 is a 'decomposition' principle in the sense required by (iii). But this point can be made more formally, by showing that fusions and remainders are 'two sides of the same coin', so to speak.

To see why, let us focus on the binary variant of fusions, sometimes called merelogical sums. Let $\psi$ be the open formula ' $z=a \vee z=b$ ', where ' $a$ ' and ' $b$ ' are terms. Then the sum of $a$ and $b$ is defined as the unique $x$ such that $F_{\psi} x$ :

(D.4) $a \sqcup b:=1 x F_{\psi} x$

Sum

In classical logic $\exists z(z=a \vee z=b)$ is always true, so A.4 implies that binary sums always exist. Is there a decomposition-correlate to this fact?

For the time being, let us introduce the placeholder $c \prec a(\operatorname{read}$ ' $c$ without $a$ ') to stand for an object that satisfies the following condition:

(1) $\quad P c(a \sqcup b)$ iff $P(c \prec a) b$

Residuation

This is a biconditional connecting composition with decomposition in a natural way; it says that $c$ is part of the sum of $a$ and $b$ iff $c$ without $a$ is part of $b$. (Note that since $a \sqcup b=b \sqcup a$, owing to the commutativity of $\vee$, we have that $P(c \prec a) b$ iff $P(c \prec b) a$.) Those familiar with logic will be reminded of a well-known residuation condition, namely

(2) $\quad p \wedge q \vdash r$ iff $p \vdash q \rightarrow r$

In classical logic, this is equivalent to

(3) $p \wedge q \vdash r$ iff $p \vdash \neg q \vee r$

from which we obtain the algebraic 'dual' of (2):

(4) $p \vdash q \vee r$ iff $p \wedge \neg q \vdash r$

This is a residuation condition in logic that is perfectly analogous to (1). 
Now, $c \longrightarrow a$ is so far just a placeholder. We want to know under what conditions (1) holds: when does $c \prec a$ exist, and what sort of thing is it? To answer the first question, we note immediately that $c \prec c$ cannot exist: it would have to be a 'null' element, an algebraic 'zero' relative to $P$, and the non-existence of such an element is a distinguishing feature of classical mereology (whence the conditional form of A.4). Thus, in (1) an exception must be made when $c=a$. Indeed, the same is true whenever $P c a$ (of which $c=a$ is a special case by A.1), since $c \prec a$ would not exist for the same reason. 'This is exactly the exclusion given in the antecedent of A.5.

To answer the second question, we now show that, for any $c$ and $a$ that meet the exclusion condition just mentioned, $c-a$ is precisely the remainder of $a$ in $c$ stipulated to exist by the relevant instance of A.5. That is, let $d$ be that remainder:

(5) $\quad \forall w(P w d \leftrightarrow(P w c \wedge \neg O w a))$

We show that, for arbitrary $b, d$ meets the constraint in (11):

$$
P c(a \sqcup b) \text { iff } P d b
$$

Proof of (6) For the right-to-left-direction, assume that $P c(a \sqcup b)$. Since $P d d$, by the $\rightarrow$-direction of (5) we have that $P d c$ and $\neg O d a$. From $P d c$ it follows immediately that $P d(a \sqcup b)$ (by A.3) and this, together with $\neg O d a$, implies that $P d b$. For the left-to-right direction, assume that $P d b$. We distinguish two cases, depending on whether or not $c$ has a part in common with $a$. In the first case, we have Oca and we want to show that $P c(a \sqcup b)$. Suppose not. Then, by A.5, $a \sqcup b$ must leave a remainder in $c$, i.e., there is some $z$ such that $\forall w(P w z \leftrightarrow(P w c \wedge \neg O w(a \sqcup b)))$. Since $P z z$ by A.1, it follows that $P z c$ and $\neg O z(a \sqcup b)$, and hence $\neg O z a$. By the $\leftarrow$-direction of (5) we obtain $P z d$ and thus, by A.3, $P z b$ (since $P d b$ by assumption). This implies $P z(a \sqcup b)$, and therefore $O z(a \sqcup b)$-contradition. So, $P c(a \sqcup b)$ after all. In the second case we have $\neg O c a$. Since we also have $P c c$ by A.1, by the $\leftarrow$-direction of (5) we obtain $P c d$. So, again by A.3, $P c b$, and hence $P c(a \sqcup b)$.

We have thus shown that the remainder of any two given objects $c$ and $a$, when it exists, meets the condition for $c \prec a$. This establishes that A.5 provides an axiomatic answer to (iii) that mirrors the answer to (ii) provided by A.4: the Remainder axiom quite simply stipulates the existence of the decomposition-correlate to (binary) composition.]

\footnotetext{
${ }^{5}$ In a boolean algebra, $x \prec y=\perp$ iff $x \prec y \leq \perp$ iff $x \leq y \sqcup \perp$ iff $x \leq y$.

${ }^{6}$ This last step assumes a principle called Filtration, which we show is a theorem of CM in $\$ 3.1$.

${ }^{7}$ Note: every boolean algebra is a residuated lattice, with $a \sqcap-b$ the residual of $\sqcap$, but it is not true in general that every residuated lattice is a boolean algebra. In the case of CM, the remainder principle serves to stipulate the existence of residuals of sums; however, merely adding a residuation axiom in place of A.5 would not be strong enough to yield classical mereology.
} 


\section{EQUIVALENCES}

It remains to be shown that $\mathrm{CM}$ is indeed an axiomatization of classical mereology. We do so by proving the equivalence of CM with two systems for classical mereology due essentially to Paul Hovda [1]. In each case we only prove one side of the equivalence, namely that the system in question is included in CM. The converse inclusion is trivial, since, as we mentioned, the five axioms of $\mathrm{CM}$ are well-known theses of classical mereology.

\subsection{PROOF 1}

In $\left[1, \$_{3.1}\right]$ it is shown that classical mereology can be axiomatized by taking A.3 and A.4 along with the following two axioms.

(A.6) $\forall x \forall y(P P x y \rightarrow \exists z(P z y \wedge \neg O z x))$

Weak Supplementation

(A.7) $\exists z \varphi \rightarrow \forall x \forall y\left(\left(P y x \wedge F_{\varphi} x\right) \rightarrow \exists z(\varphi \wedge O z y)\right)$

Filtration

Call this system $\mathrm{CM} 1$. We show that $\mathrm{CM}_{1} \subseteq \mathrm{CM}$. Specifically, first we show that A.6 is entailed by A.1 + A.2 + A.5. Next we show that A.7 is entailed by A.1 + A.2 + A.3 + A.5.

SteP 1: Proof of A.6 Assume for conditional proof that PPxy. By D.1 we have that $x \neq y$ and $P x y$. So, by Antisymmetry, $\neg P y x$. Hence, by Remainder, there is some $z$ such that $\forall w(P w z \leftrightarrow(P w y \wedge \neg O w x))$. By Reflexivity, $P z z$, and hence $P z y \wedge \neg O z x$ as required.

STEP 2: Proof of A.7 Assume the antecedent of A.7 and assume, for arbitrary $x$, $y$, and $\varphi$, that $P y x$ and $F_{\varphi} x$. We need to show that $\exists z(\varphi \wedge O z y)$. We distinguish two cases, depending on whether or not $x=y$. In case $x=y$, we have by assumption that $F_{\varphi} y$ and hence, by D.3, $\forall z(\varphi \rightarrow P z y)$. By assumption we also have $\exists z \varphi$ and so we obtain $\exists z(\varphi \wedge P z y)$, whence $\exists z(\varphi \wedge O z y)$ by Reflexivity and D.2. In the second case, where $x \neq y$, we immediately have $P P y x$ by D.1, hence $\neg P y x$ by Antisymmetry. By Remainder, this implies that there's some $c$ satisfying the following condition:

(7) $\quad \forall w(P w c \leftrightarrow(P w x \wedge \neg O w y))$

\footnotetext{
${ }^{8}$ Actually, the formulation of A.7 in [1] is missing the antecedent. That formulation is too strong, admitting instances that are false in any one-element model in which the only element of the domain fails to satisfy $\phi_{x}$. The error is corrected in $[5, \$ 2]$.
} 
Suppose for reductio that $\forall z(\varphi \rightarrow \neg O z y)$. Let $a$ be some arbitrary object satisfying $\varphi$ (whose existence follows from the assumption that $\exists z \varphi$ ). Since $x$ is the fusion of the $\varphi$ s (again by assumption), D.3 implies that Pax. And since we also have $\neg$ Oay, we obtain $P a c$ by the $\leftarrow$-direction of $(\nabla)$. But recall that $a$ was arbitrary, hence $\forall z(\varphi \rightarrow P z c)$, which is to say that $c$ is an upper bound of the $\varphi$ s. Thus, since $x$ is the least upper bound of the $\varphi$ s, it follows by D.3 that Pxc. Now recall that $P y x$, so by Transitivity Pyc. By the $\rightarrow$-direction of $(\nabla)$, this implies that $P y x \wedge \neg O y y$. But Reflexivity gives us $P y y$, and so we obtain $O y y$. Contradiction. It follows that $\neg \forall z(\varphi \rightarrow \neg O z y)$, i.e., $\exists z(\varphi \wedge O z y)$.

\subsection{PROOF 2}

In $\left[1, \$_{4}\right]$ it is shown that classical mereology can also be axiomatized by taking A.2, A.3 and A.4 together with the following axioms:"

(A.8) $\forall x(\neg 1 x \rightarrow \exists z(\neg O z x \wedge \forall w((\neg O w x \rightarrow P w z) \wedge(\neg O w z \rightarrow P w x))))$ Comp

(A.9) $\exists x \exists y y \neq x \rightarrow \neg \exists x \forall y P x y$

No Zero

where

(D.5) $1 x: \equiv \forall y P y x$

Universe

We show again that this system, $\mathrm{CM}_{2}$, is included in $\mathrm{CM}$, i.e., that A.8 and A.9 follow from our axioms A.1 A.5.

SteP 1: Proof of A.8 Notice that, by A.4, there is a fusion of those objects that satisfy $\exists y z=y$, namely a fusion of everything. Call it $T$. By the first conjunct of D.3, everything is part of $T$, so such a fusion satisfies the predicate defined in D.5. Now, the following is an instance of A.5:

(8) $\quad \forall y(\neg P \top y \rightarrow \exists z \forall w(P w z \leftrightarrow(P w \top \wedge \neg O w y)))$

Thus, since PTT by A.1, we obtain:

(9) $\forall x(\neg 1 x \rightarrow \exists z \forall w(P w z \leftrightarrow(P w \top \wedge \neg O w x)))$

Let $a$ be some non- 1 individual and let $b$ be a matching witness for $z$ in (9). We want to show that $b$ is also a witness for $z$ in the corresponding instance of A.8. We have to prove three claims:

(a) $\neg \mathrm{Oba}$

\footnotetext{
${ }^{9}$ Strictly speaking, the formulation of A.8 in $[1, \$ 4]$ uses a stronger variant of $O$ that rules out overlap by the null element. However, the difference is immaterial; see [1, pp. 76, 82].
} 
(b) $\forall w(\neg O w a \rightarrow P w b)$

(c) $\forall w(\neg O w b \rightarrow P w a)$

By (9) we have that $\forall w(P w b \leftrightarrow(P w \top \wedge \neg O w a))$, and since $P w \top$ holds for all $w$ by definition, we obtain

(10) $\forall w(P w b \leftrightarrow \neg O w a)$

To prove (a), suppose for reductio that $\mathrm{Oba}$. Then, for some $c, P c b \wedge P c a$. From the first conjunct, together with the $\rightarrow$-direction of (10), we obtain $\neg O c a$. But this contradicts the second conjunct, $P c a$ (since $P c c$ by A.1). So $\neg O b a$. Concerning (b), we note that this claim is just the $\leftarrow$-direction of (10). As for (c), pick arbitrary $w$ so that $\neg O w b$. We want to show that $P w a$. Suppose for reductio that $\neg P w a$. By A.5, there must be some remainder of $a$ in $w$, i.e., some $c$ such that $\forall y(P y c \leftrightarrow$ $(P y w \wedge \neg O y a)$ ). By A.1, $P c c$, and so $P c w \wedge \neg O c a$. But then the $\rightarrow$-direction of (10) gives us $P c b$, and hence $c$ is part of both $w$ and $b$. So $O w b$. This contradicts our initial assumption on $w$, and so we conclude that $P w a$.

Step 2: Proof of A.9 Assume according to the antecedent of A.9 that there are at least two things, $a$ and $b$. Without loss of generality, let $\neg P a b$ (since if $P a b$, we could assume $\neg P b a$ by A.2 and reason mutatis mutandis). By A.5, we have for some $c$ that $\forall w(P w c \leftrightarrow(P w a \wedge \neg O w b))$. Now suppose there were an object-call it $\perp-$ so that $\forall w P \perp w$. Then we would have $P \perp c$, and hence $P \perp a \wedge \neg O \perp b$. But by A.1 the latter conjunct contradicts $P \perp b$, which holds by supposition. Hence, there can be no such $\perp$.

\section{FINAL REMARKS}

It should be noted that Step 2 in the last proof shows that A.9 is actually redundant in $\mathrm{CM}_{2}$, since it turns out that A.4 and A.8 imply both A.1 and A.5. For A.1, see $[1$, p. 75]. For A.5, given arbitrary $a$ and $b$ such that $\neg P a b$, we always get a remainder of $b$ in $a$ by taking the complement of $b$ if $1 a$, and otherwise the complement of the fusion of $b$ with $a$ 's complement (where, generally speaking, the complement of $x$ is the entity $z$ posited by the consequent of A.8, whose uniqueness follows from A.2). Indeed, since A.1 follows from A.8 alone, the latter axiom is enough to derive A.9 as a theorem, as noted in $\left[5, \S_{3}\right]$ : if $\perp$ existed, it would overlap everything, hence it could not satisfy the first conjunct in the existential consequent of A.8.

Does this mean that our axiom system is just a minor variant of $\mathrm{CM}_{2}$, i.e., of A.2 + A.3 + A.4 + A.8? In a way, yes: we are just splitting A.8 into A.1 and A.5. Philosophically, however, the difference is significant. 
For one thing, the very fact that it implies Reflexivity shows that A.8 is not just a decomposition principle; it answers part of question (i) along with question (iii). ${ }^{\text {TO }}$ In our axiom system, by contrast, these questions are answered separately. (That A.1 does not follow from A.5 can be seen by considering a model with a single element, $a$, such that $\neg P a a$.)

Secondly, A.8 is not just a decomposition principle, for it is not just about how a given entity may be decomposed; it actually stipulates the existence of something outside that entity. Since there are things that are not part of, say, this table, according to A.8 there is some humongous composite entity made up of absolutely everything that doesn't overlap this table. Since there is more to this world than Ohio, there is something that consists of everything except Ohio. Entities of this sort-the table's complement, Ohio's complement-will not be objectionable to a classical mereologist who accepts the Unrestricted Fusion axiom A.4. That axiom, however, is intended to answer question (ii), and so again it appears that A.8 oversteps its bounds as an answer to (iii). By contrast, A.5 is truly a decomposition principle in that it tells us one critical way in which any object $x$ may be decomposed. Provided $\neg P x y, x$ may be decomposed entirely into $x \prec y$ and (when it exists) $x \prec(x \prec y)$. That is, A.5 only stipulates the existence of parts of $x .{ }^{\text {T }}$

For these reasons, while registering the formal similarity between $\mathrm{CM}$ and $\mathrm{CM}_{2}$, we conclude that the two systems differ significantly vis à vis the general task of answering questions (i)-(iii). The axiomatic answers provided by $\mathrm{CM}_{2}$, like those of $\mathrm{CM} 1$ and of other standard axiomatizations of classical mereology, are to some extent indirect; those provided by CM are explicit and neatly separated. They are, in this sense, the 'natural' answers we were looking for. ${ }^{\mathrm{M}}$

\section{REFERENCES}

[1] Hovda, P., "What is classical mereology?", Journal of Philosophical Logic, 38 (2009), pp. 55-82.

[2] Leonard, H.S., and N. Goodman, "The calculus of individuals and its uses", Journal of Symbolic Logic, 5 (1940), pp. 45-55.

[3] Leśniewski, S., Podstawy ogólnej teoryi mnogości. I, Moskow, Prace Polskiego Koła Naukowego w Moskwie, Sekcya matematyczno-przyrodnicza, 1916;

\footnotetext{
${ }^{10}$ In this regard, $\mathrm{CM}_{2}$ is more like $\mathrm{CM}_{1}$, where both Reflexivity and Antisymmetry come from the other axioms [1, p. 67].

${ }^{11}$ Of course, there is a sense in which the existence of such parts still bleeds into the territory of composition. For the remainder of $y$ in $x$ is not just a part of $x$; it is a part composed of parts, viz. those parts of $x$ that are disjoint from $y$. However, this is just a way of saying that A.5 tells us the exact mereological make-up of the remainder, and that is a way of answering question (iii).

${ }^{12}$ Thanks to Paul Hovda and Sheiva Kleinschmidt for discussion. The research and writing of this paper was supported by a 2017-2018 Leverhulme Research Fellowship from the Leverhulme Trust.
} 
Eng. trans. by D. I. Barnett: "Foundations of the general theory of sets. I", in S. Leśniewski, Collected Works, ed. by S. J. Surma et al., Dordrecht, Kluwer Academic Publishers, 1991, vol. 1, pp. 129-173.

[4] Simons, P. M., Parts. A Study in Ontology, Oxford, Clarendon Press, 1987.

[5] Varzi, A. C., 'On three axiom systems for classical mereology', Logic and Logical Philosophy, in press. 